\title{
Mathematical Modelling of Radiative Hydromagnetic Thermosolutal Nanofluid Convection Slip Flow in Saturated Porous Media
}

\author{
Mohammed Jashim Uddin, ${ }^{1}$ Osman Anwar Bég, ${ }^{2}$ and Ahmad Izani Md. Ismail ${ }^{3}$ \\ ${ }^{1}$ American International University-Bangladesh, Banani, Dhaka 1213, Bangladesh \\ ${ }^{2}$ Gort Engovation Research (Biomechanics), Southmere Avenue, Bradford BD73NU, UK \\ ${ }^{3}$ Universiti Sains Malaysia, Penang 11800, Malaysia \\ Correspondence should be addressed to Mohammed Jashim Uddin; jashim_74@yahoo.com
}

Received 5 January 2014; Revised 5 March 2014; Accepted 14 April 2014; Published 13 May 2014

Academic Editor: Kuppalapalle Vajravelu

Copyright (C) 2014 Mohammed Jashim Uddin et al. This is an open access article distributed under the Creative Commons Attribution License, which permits unrestricted use, distribution, and reproduction in any medium, provided the original work is properly cited.

\begin{abstract}
High temperature thermal processing of nanomaterials is an active area of research. Many techniques are being investigated to manipulate properties of nanomaterials for medical implementation. In this paper, we investigate thermal radiation processing of a nanomaterial fluid sheet extruded in porous media. A mathematical model is developed using a Darcy drag force model. Instead of using linear radiative heat flux, the nonlinear radiative heat flux in the Rosseland approximation is taken into account which makes the present study more meaningful and practically useful. Velocity slip and thermal and mass convective boundary conditions are incorporated in the model. The Buongiornio nanofluid model is adopted wherein Brownian motion and thermophoresis effects are present. The boundary layer conservation equations are transformed using appropriate similarity variables and the resulting nonlinear boundary value problem is solved using Maple 14 which uses the Runge-Kutta-Fehlberg fourth fifth order numerical method. Solutions are validated with previous nonmagnetic and nonradiative computations from the literature, demonstrating excellent agreement. The influence of Darcy number, magnetic field parameter, hydrodynamic slip parameter, convection-conduction parameter, convection-diffusion parameter, and conduction-radiation parameter on the dimensionless velocity, temperature, and nanoparticle concentration fields is examined in detail. Interesting patterns of relevance are observed to improve manufacturing of nanofluids.
\end{abstract}

\section{Introduction}

Nanofluid transport in porous media has developed into a substantial area of research in recent years. This has been motivated by the thermally enhancing properties of nanofluids [1] which are achieved owing to the presence of metallic nanoparticles suspended in base fluids (water, oil, etc.). Recent applications of nanofluid convection in porous media include solar collectors [2,3], microbial fuel cells [4], materials processing [5, 6], biological propulsion [7], and geothermal energy systems [8] (where nanofluid injection results in greater thermal efficiency). These investigations have built on earlier seminal theoretical works of Buongiornio [9] and Nield and Kuznetsov [10] wherein elegant formulations for nanofluid convection have been developed and which prioritize Brownian motion and thermophoresis effects. Electrically conducting nanofluid flows which respond to the imposition of magnetic fields have also received some attention in recent years motivated by manufacture of complex fluids for aerospace and industrial systems [11-14]. In various manufacturing processes, high temperature effects and also porous media are also encountered, in some cases, simultaneously. Thermal radiation heat transfer [15] is important in the former. Drag forces induced by the porous material fibers exert a significant influence on fluid flow and heat transfer characteristics in the latter. Numerous studies of radiativeconvective flows in porous media have been communicated (e.g., [16]), and are relevant also to thermal insulation engineering and materials fabrication among other technologies. Interesting studies in this regard include Vafai and Tien [17], 
Takhar et al. [18], and Rashidi et al. [19]. In these studies generally algebraic flux models have been used to solve for the radiative contribution to heat transfer and this is generally simulated via a conduction-radiation parameter. The Rosseland diffusion, non-gray Schuster-Schwartzchild twoflux and other models have been employed by the researchers. The Rosseland model is the easiest to implement and allows radiative effects to be studied via a single dimensionless parameter, for example, Rosseland number. In the context of porous media studies, the Darcy drag force model which represents the bulk matrix effect on fluid transport is the most popular although it is limited to low-speed viscousdominated flows. Numerous articles have utilized this model for porous media nanofluid modeling including Nield and Kuznetsov [20], Uddin et al. [21], Hady et al. [22], and others [23-26].

The objective of the present investigation is to extend the work of Makinde and Aziz [27] for MHD Navier slip flow of a nanofluid in porous media over a radiating stretching sheet in the presence of thermal and mass convective boundary conditions. This problem is of interest in nanomaterial manufacturing processes. Slip effects have been shown to be important in stretching sheet flows and recent studies include Hamad et al. [28] and Prasad et al. [29-31]. The governing partial differential boundary layer equations are reduced to a two-point boundary value problem with the aid of appropriate similarity variables. The reduced equations have been numerically solved by the use of an efficient Runge-Kutta-Fehlberg fourth fifth order numerical method which is available in Maple 14 [32]. The effects of key thermophysical parameters on the fluid velocity, temperature, and nanoparticle volume fraction (concentration) have been examined in detail. Validation of Maple solutions is included using earlier published results for the nonradiative case as examined by Dayyan et al. [33]. The current study has not been communicated in the scientific literature to the best of the authors' knowledge.

\section{Governing Nanofluid Transport Model}

Consider a two-dimensional regime with a coordinate system with the $\bar{x}$-axis aligned horizontally and the $\bar{y}$-axis is normal to it. A transverse magnetic field $B_{0}$ acts normal to the bounding surface. The magnetic Reynolds number is small so that the induced magnetic field is effectively negligible when compared to the applied magnetic field. We neglect the electric field associated with the polarization of charges and Hall effects. It is further assumed that the left of the plate is heated by the convection from the hot fluid of temperature $T_{f}\left(>T_{w}>T_{\infty}\right)$ which provides a variable heat transfer coefficient $h_{f}(\bar{x})$. Consequently a thermal convective boundary condition arises. It is further assumed that the concentration in the left of the plate $C_{f}$ is higher than that of the plate concentration $C_{w}$ and free stream concentration $C_{\infty}$ which provides a variable mass transfer coefficient $h_{m}(\bar{x})$. As a result a mass convective boundary condition arises. The Oberbeck-Boussinesq approximation is utilized and the four field equations are the conservation of mass, momentum, thermal energy, and the nanoparticles volume fraction. These equations can be written in terms of dimensional forms, extending the formulations of Buongiorno [9] and Makinde and Aziz [27]:

$$
\begin{gathered}
\frac{\partial \bar{u}}{\partial \bar{x}}+\frac{\partial \bar{v}}{\partial \bar{y}}=0 \\
\rho_{f}\left(\bar{u} \frac{\partial \bar{u}}{\partial \bar{x}}+\bar{v} \frac{\partial \bar{u}}{\partial \bar{y}}\right)=\mu \frac{\partial^{2} \bar{u}}{\partial \bar{y}^{2}}-\frac{\mu}{K_{P}} \bar{u}-\sigma B_{0}^{2} \bar{u} \\
\bar{u} \frac{\partial T}{\partial \bar{x}}+\bar{v} \frac{\partial T}{\partial \bar{y}}=\alpha \frac{\partial^{2} T}{\partial \bar{y}^{2}}+\tau\left\{D_{B} \frac{\partial C}{\partial \bar{y}} \frac{\partial T}{\partial \bar{y}}+\left(\frac{D_{T}}{T_{\infty}}\right)\left(\frac{\partial T}{\partial \bar{y}}\right)^{2}\right\} \\
-\frac{1}{\rho_{f} c_{f}} \frac{\partial q_{r}}{\partial \bar{y}}, \\
\bar{u} \frac{\partial C}{\partial \bar{x}}+\bar{v} \frac{\partial C}{\partial \bar{y}}=D_{B} \frac{\partial^{2} C}{\partial \bar{y}^{2}}+\left(\frac{D_{T}}{T_{\infty}}\right) \frac{\partial^{2} T}{\partial \bar{y}^{2}}
\end{gathered}
$$

The appropriate boundary conditions are, following Datta [34] and Karniadakis et al. [35],

$$
\begin{gathered}
\bar{u}=\bar{u}_{w}+\bar{u}_{\text {slip }}, \quad \bar{v}=0, \\
-k \frac{\partial T}{\partial \bar{y}}=h_{f}\left(T_{f}-T\right), \quad-D_{B} \frac{\partial C}{\partial \bar{y}}=h_{m}\left(C_{f}-C\right) \\
\text { at } \bar{y}=0, \\
\bar{u} \longrightarrow 0, \quad T \longrightarrow T_{\infty}, \quad C \longrightarrow C_{\infty} \quad \text { as } \bar{y} \longrightarrow \infty .
\end{gathered}
$$

Here $\alpha=k /(\rho c)_{f}$ : thermal diffusivity of the fluid, $\tau=$ $(\rho c)_{p} /(\rho c)_{f}$ : ratio of heat capacity of the nanoparticle and fluid, $K_{p}$ : permeability of the medium, $(\bar{u}, \bar{v})$ : velocity components along $\bar{x}$ and $\bar{y}$ axes, $\bar{u}_{w}=U_{r}(\bar{x} / L)$ : velocity of the plate, $L$ : characteristic length of the plate, $\bar{u}_{\text {slip }}=N_{1} \nu(\partial \bar{u} / \partial \bar{y})$ : linear slip velocity, $N_{1}$ : velocity slip factor with dimension $\mathrm{s} / \mathrm{m}, \rho_{f}$ : density of the base fluid, $\sigma$ : electric conductivity, $\mu$ : dynamic viscosity of the base fluid, $\rho_{P}$ : density of the nanoparticles, $\left(\rho C_{P}\right)_{f}$ : effective heat capacity of the fluid, $\left(\rho C_{P}\right)_{P}$ : effective heat capacity of the nanoparticle material, $\varepsilon$ : porosity, $D_{B}$ : Brownian diffusion coefficient, $D_{T}$ : thermophoretic diffusion coefficient, and $q_{r}$ : radiative heat transfer in $\bar{y}$-direction. We consider the fluid to be a gray, absorbing-emitting but nonscattering medium. We also assume that the boundary layer is optically thick and the Rosseland approximation or diffusion approximation for radiation is valid [36, 37]. Thus, the radiative heat flux for an optically thick boundary layer (with intensive absorption), as elaborated by Sparrow and Cess [38], is defined as $q_{r}=-\left(4 \sigma_{1} / 3 k_{1}\right)\left(\partial T^{4} / \partial \bar{y}\right)$, where $\sigma_{1}$ $\left(=5.67 \times 10^{-8} \mathrm{~W} / \mathrm{m}^{2} \mathrm{~K}^{4}\right)$ is the Stefan-Boltzmann constant and $k_{1}\left(\mathrm{~m}^{-1}\right)$ is the Rosseland mean absorption coefficient. Purely analytical solutions to the partial differential boundary value problem defined by (1)-(3) are not possible. Even a numerical solution is challenging. Hence we aim to transform 
the problem to a system of ordinary differential equations. We define the following dimensionless transformation variables:

$$
\begin{gathered}
\eta=\frac{\bar{y}}{\sqrt{K_{p}}}, \quad \psi=U_{r} \frac{\bar{x}}{L} \sqrt{K_{p}} f(\eta), \\
\theta(\eta)=\frac{T-T_{\infty}}{T_{f}-T_{\infty}}, \quad \phi(\eta)=\frac{C-C_{\infty}}{C_{f}-C_{\infty}},
\end{gathered}
$$

where $L$ is the characteristic length. From (4), we have $T=$ $T_{\infty}\left\{1+\left(T_{r}-1\right) \theta\right\}$, where $T_{r}=T_{f} / T_{\infty}$ (the wall temperature excess ratio parameter) and hence $T^{4}=T_{\infty}^{4}\left\{1+\left(T_{r}-1\right) \theta\right\}^{4}$. Substitution of (4) into (2)-(3) generates the following similarity equations:

$$
\begin{gathered}
f^{\prime \prime \prime}+\operatorname{ReDa}\left(f f^{\prime \prime}-f^{\prime 2}-M f^{\prime}\right)-f^{\prime}=0, \\
\theta^{\prime \prime}+\operatorname{RePrDa} f \theta^{\prime}+\operatorname{Pr}\left[\mathrm{Nb} \theta^{\prime} \phi^{\prime}+\mathrm{Nt} \theta^{\prime 2}\right] \\
+\frac{4}{3 R}\left[\left\{1+\left(T_{r}-1\right) \theta\right\}^{3} \theta^{\prime}\right]^{\prime}=0, \\
\phi^{\prime \prime}+\operatorname{LeReDa} f \phi^{\prime}+\frac{\mathrm{Nt}}{\mathrm{Nb}} \theta^{\prime \prime}=0 .
\end{gathered}
$$

The relevant boundary conditions are

$$
\begin{gathered}
f(0)=0, \quad f^{\prime}(0)=1+a f^{\prime \prime}(0), \\
\theta^{\prime}(0)=-\mathrm{Nc}[1-\theta(0)], \quad \phi^{\prime}(0)=-\mathrm{Nd}[1-\phi(0)], \\
f^{\prime}(\infty)=\theta(\infty)=\phi(\infty)=0,
\end{gathered}
$$

where primes denote differentiation with respect to $\eta$. The thermophysical dimensionless parameters arising in (5)-(8) are defined as follows: $\operatorname{Re}=U_{r} L / \nu$ is the Reynolds number, $\mathrm{Da}=K_{p} / L^{2}$ is the Darcy number, $M=\sigma B_{0}^{2} L / U_{r} \rho$ is the magnetic field parameter, $\operatorname{Pr}=\nu / \alpha$ is the Prandtl number, $\mathrm{Nt}=\tau D_{T}\left(T_{f}-T_{\infty}\right) / \nu T_{\infty}$ is the thermophoresis parameter, $\mathrm{Nb}=\tau D_{B}\left(C_{f}-C_{\infty}\right) / \nu$ is the Brownian motion parameter, $R=k k_{1} / 4 \sigma_{1} T_{\infty}^{3}$ is the convection-radiation parameter, Le = $v / D_{B}$ is the Lewis number, $a=N_{1} v / \sqrt{K_{P}}$ is the hydrodynamic (momentum) slip parameter, $\mathrm{Nd}=h_{m} \sqrt{K_{P}} / D_{B}$ is the convection-diffusion parameter, and $\mathrm{Nc}=h_{f} \sqrt{K_{P}} / k$ is the convection-conduction parameter.

Quantities of physical interest are the local friction factor, $C_{f \bar{x}}$, the local Nusselt number, $\mathrm{Nu}_{\bar{x}}$, and the local Sherwood number, $\mathrm{Sh}_{\bar{x}}$. Physically, $C_{f x}$ represents the wall shear stress, $\mathrm{Nu}_{\bar{x}}$ defines the heat transfer rates, and $\mathrm{Sh}_{\bar{x}}$ defines the mass transfer rates:

$$
\begin{gathered}
C_{f x} \operatorname{Re}_{x}^{-1} \mathrm{Da}_{x}^{0.5}=2 f^{\prime \prime}(0) \\
\mathrm{Nu}_{\bar{x}} \mathrm{Da}_{\bar{x}}^{0.5}=-\left[1+\frac{4}{3 R}\left\{1+\left(T_{r}-1\right) \theta(0)\right\}^{3}\right] \theta^{\prime}(0), \\
\operatorname{Sh}_{\bar{x}} \mathrm{Da}_{\bar{x}}^{0.5}=-\phi^{\prime}(0)
\end{gathered}
$$

where $\mathrm{Da}_{\bar{x}}=K_{p} / \bar{x}^{2}$ is the local Darcy number for Darcian porous media and $\operatorname{Re}_{\bar{x}}=\bar{u}_{w} \bar{x} / \nu$ is the local Reynolds number.
We note that, for purely hydromagnetic boundary layer $(M=$ 0 ) and no slip boundary condition $(a=0)$, the problem reduces to the problem which has been recently considered and investigated by Dayyan et al. [33] when $\mathrm{Nc}=\mathrm{Nd}=R \rightarrow$ $\infty, \mathrm{Da}=1, \mathrm{Nt}=0$, and $\mathrm{Nb} \rightarrow 0$ in our model and $n=0$ in their paper. This provides a useful benchmark for validating the present model.

\section{Numerical Solutions}

The set of nonlinear ordinary differential equations (5)-(7) subject to the boundary conditions in (8) have been solved numerically using Maple dsolve command with numeric option. This software uses the Runge-Kutta-Fehlberg fourth fifth (RKF45) order numerical method for solving twopoint boundary value problem. The Runge-Kutta-Fehlberg fourth fifth order numerical method is a well-established adaptive numerical method for solving system of ordinary differential equations with associated conditions. The RungeKutta-Fehlberg algorithm uses both a fifth and a fourth order Runge-Kutta. The error of this algorithm is determined by subtracting these two values and can be used for adaptive step sizing. The formula for the fifth fourth order Runge-KuttaFehlberg algorithm is given below:

$$
\begin{aligned}
& k_{0}= f\left(x_{i}, y_{i}\right), \\
& k_{1}=f\left(x_{i}+\frac{1}{4} h, y_{i}+\frac{1}{4} h k_{0}\right), \\
& k_{2}=f\left(x_{i}+\frac{3}{8} h, y_{i}+\left(\frac{3}{32} k_{0}+\frac{9}{32} k_{1}\right) h\right), \\
& k_{3}=f\left(x_{i}+\frac{12}{13} h, y_{i}+\left(\frac{1932}{2197} k_{0}-\frac{7200}{2197} k_{1}+\frac{7296}{2197} k_{2}\right) h\right), \\
& k_{4}=f\left(x_{i}+h, y_{i}+\left(\frac{439}{216} k_{0}-8 k_{1}+\frac{3860}{513} k_{2}-\frac{845}{4104} k_{3}\right) h\right), \\
& k_{5}=f\left(x_{i}+\frac{1}{2} h, y_{i}\right. \\
&\left.\quad+\left(-\frac{8}{27} k_{0}+2 k_{1}-\frac{3544}{2565} k_{2}+\frac{1859}{4104} k_{3}-\frac{11}{40} k_{4}\right) h\right), \\
& y_{i+1}=y_{i}+\left(\frac{25}{216} k_{0}+\frac{1408}{2565} k_{2}+\frac{2197}{4104} k_{3}-\frac{1}{5} k_{4}\right) h, \\
& z_{i+1}=z_{i} \\
&+\left(\frac{16}{135} k_{0}+\frac{6656}{12825} k_{2}+\frac{28561}{56430} k_{3}-\frac{9}{50} k_{4}+\frac{2}{55} k_{5}\right) h,
\end{aligned}
$$

where $y$ is a fourth-order Runge-Kutta and $z$ is a fifth-order Runge-Kutta. An estimate of the error can be obtained by subtracting the two values obtained. If the error exceeds a specified threshold, the results can be recalculated using a smaller step size. The approach to estimating the new step size is shown below:

$$
h_{\text {new }}=h_{\text {old }}\left(\frac{\varepsilon h_{\text {old }}}{2\left|z_{i+1}-y_{i+1}\right|}\right)^{1 / 4} .
$$


TABLE 1: Comparison of $f(\eta), f^{\prime}(\eta)$, and $\theta(\eta)$ when $\mathrm{Da}=1$.

\begin{tabular}{|c|c|c|c|c|c|c|}
\hline \multirow{2}{*}{$\eta$} & \multicolumn{2}{|c|}{$f(\eta)$} & \multicolumn{2}{|c|}{$f^{\prime}(\eta)$} & \multicolumn{2}{|c|}{$\theta(\eta)$} \\
\hline & $\begin{array}{c}\text { Dayyan et al. [33] } \\
\text { (HAM) }\end{array}$ & $\begin{array}{c}\text { Present } \\
\text { (Maple RKF45) }\end{array}$ & $\begin{array}{c}\text { Dayyan et al. [33] } \\
\text { (HAM) }\end{array}$ & $\begin{array}{c}\text { Present } \\
\text { (Maple RKF45) }\end{array}$ & $\begin{array}{c}\text { Dayyan et al. [33] } \\
\text { (HAM) }\end{array}$ & $\begin{array}{c}\text { Present } \\
\text { (Maple RKF45) }\end{array}$ \\
\hline 0.0 & 0.00000 & 0.00000 & 0.99999 & 0.99999 & 0.99999 & 0.99999 \\
\hline 0.2 & 0.17042 & 0.17420 & 0.75376 & 0.75364 & 0.89633 & 0.90037 \\
\hline 0.4 & 0.30564 & 0.30549 & 0.56813 & 0.56797 & 0.80205 & 0.80408 \\
\hline 0.6 & 0.40571 & 0.40443 & 0.42820 & 0.42804 & 0.71301 & 0.71343 \\
\hline 0.8 & 0.47505 & 0.47900 & 0.32272 & 0.32259 & 0.62792 & 0.6297 \\
\hline 1.0 & 0.53802 & 0.53519 & 0.24322 & 0.24312 & 0.55226 & 0.55375 \\
\hline 1.2 & 0.58010 & 0.57755 & 0.18330 & 0.18322 & 0.48134 & 0.48541 \\
\hline 1.4 & 0.61161 & 0.60947 & 0.13814 & 0.13808 & 0.42056 & 0.42451 \\
\hline 1.6 & 0.64096 & 0.63352 & 0.10410 & 0.10406 & 0.36595 & 0.37059 \\
\hline 1.8 & 0.65374 & 0.65165 & 0.07845 & 0.07843 & 0.31817 & 0.32307 \\
\hline 2.0 & 0.66952 & 0.66531 & 0.05912 & 0.05911 & 0.27657 & 0.28137 \\
\hline
\end{tabular}

TABle 2: Comparison of Skin friction factor $\left(-f^{\prime \prime}(0)\right)$ for several Reynolds numbers when $\mathrm{Da}=1, a=M=0$, and $R \rightarrow \infty$.

\begin{tabular}{lccc}
\hline \multirow{2}{*}{ Re } & \multicolumn{2}{c}{ Dayyan et al. [33] } & Present results \\
& RK & HAM & Maple RKF45 \\
\hline 1 & 1.4242 & 1.4198 & 1.4198 \\
1.5 & 1.5811 & 1.5799 & 1.5808 \\
2 & 1.7320 & 1.7234 & 1.7319 \\
5 & 2.4494 & 2.4394 & 2.4492 \\
\hline
\end{tabular}

TABLE 3: Comparison between RKF45, HAM, and RK for the values of heat transfer rate $\left(-\theta^{\prime}(0)\right)$ for several values of Reynolds number when $\mathrm{Nc}=\mathrm{Nd}=R \rightarrow \infty$ and $\mathrm{Pr}=\mathrm{Da}=1$.

\begin{tabular}{lccc}
\hline \multirow{2}{*}{ Re } & \multicolumn{2}{c}{ Dayyan et al. [33] } & Present result \\
& RK & HAM & Maple RKF45 \\
\hline 1 & 0.5033 & 0.5030 & 0.5038 \\
1.5 & 0.6422 & 0.6456 & 0.6430 \\
2 & 0.7592 & 0.7518 & 0.7539 \\
5 & 1.2576 & 1.2636 & 1.2551 \\
\hline
\end{tabular}

The step size is taken as $\Delta \eta=0.001$ and the convergence criterion was set to $10^{-6}$. The asymptotic boundary conditions given by (8) were replaced by using a value of 10 for the similarity variable $\eta_{\max }$ as follows:

$$
\eta_{\max }=10, \quad f^{\prime}(10)=\theta(10)=\phi(\infty)=0 .
$$

The choice of $\eta_{\max }=10$ ensured that all numerical solutions approached the asymptotic values in correct manner. The compilation times of this algorithm are of the order of several minutes on personal computer.

\section{Results and Discussion}

To check the accuracy of the present Maple code, comparisons of the skin friction factor and heat transfer rate have been conducted with published results obtained by the Runge-Kutta and homotopy analysis methods. The comparisons are presented in Tables 1, 2, and 3. Very good agreement

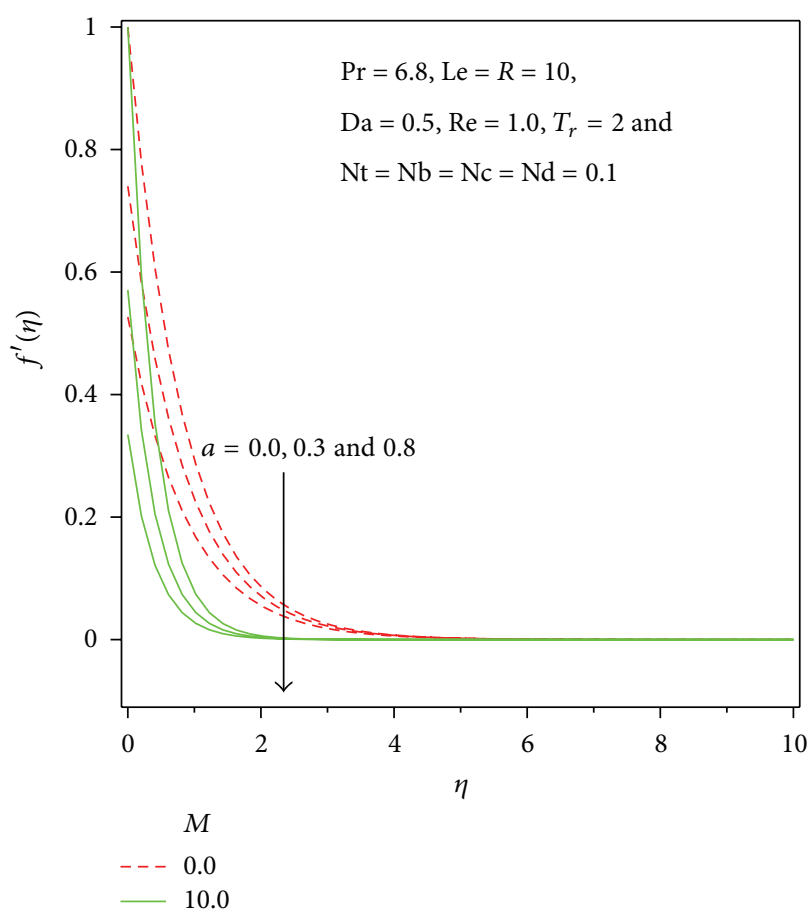

FIGURE 1: Effect of hydrodynamic slip on velocity for various magnetic parameters.

between the Maple code and the other results confirms the accuracy of the method used.

In Figures 1-10, we have examined only magnetic field, hydrodynamic slip, conduction-radiation, convection-conduction, and convection-diffusion effects on the heat, mass, and momentum transfer characteristics. The nanofluid (thermophoresis, Brownian motion), Lewis number, and Prandtl number effects are well known and have been elucidated in detail in other studies (see, for example, Uddin et al. [5]). For brevity we consider only specific parameter effects. Reynolds number $(\mathrm{Re})$ is fixed at unity and this is valid for low-speed Darcian transport. 

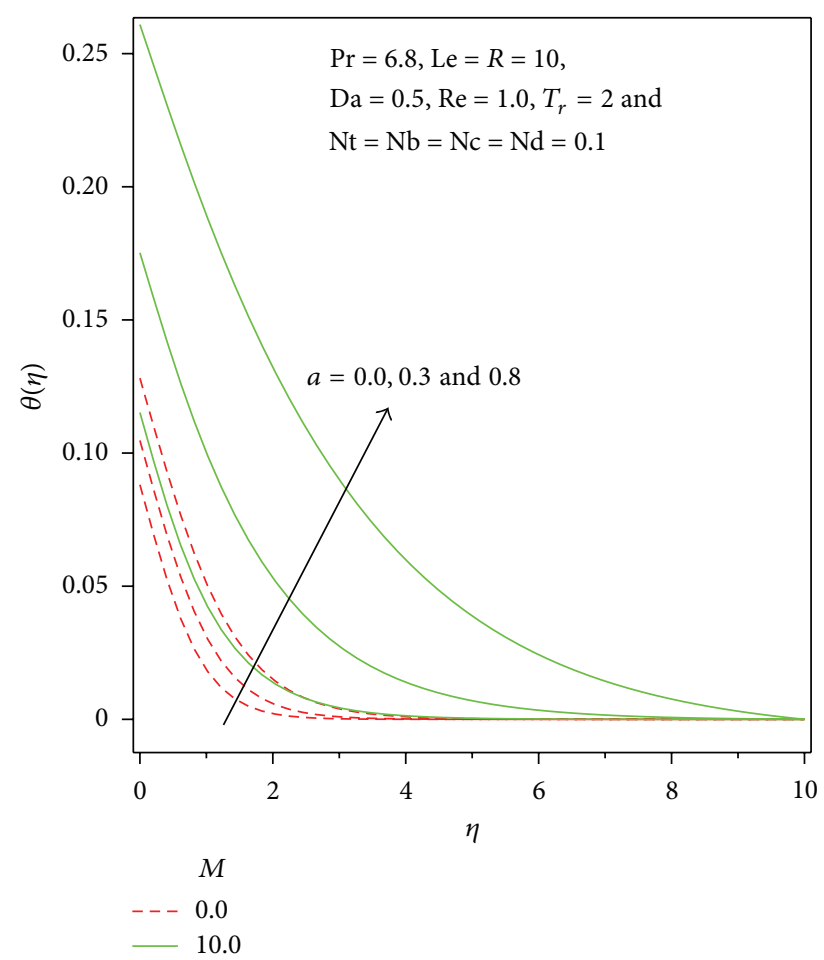

FIGURE 2: Effect of hydrodynamic slip on temperature for various magnetic parameters.
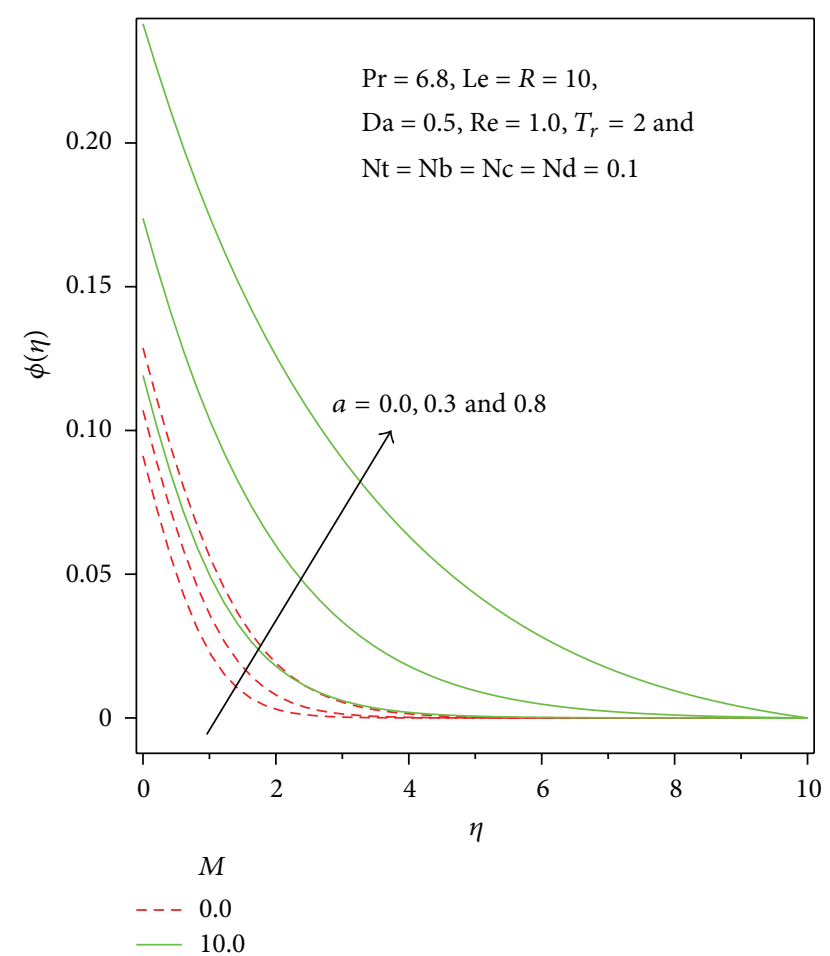

FIGURE 3: Effect of hydrodynamic slip on concentration for various magnetic parameters.

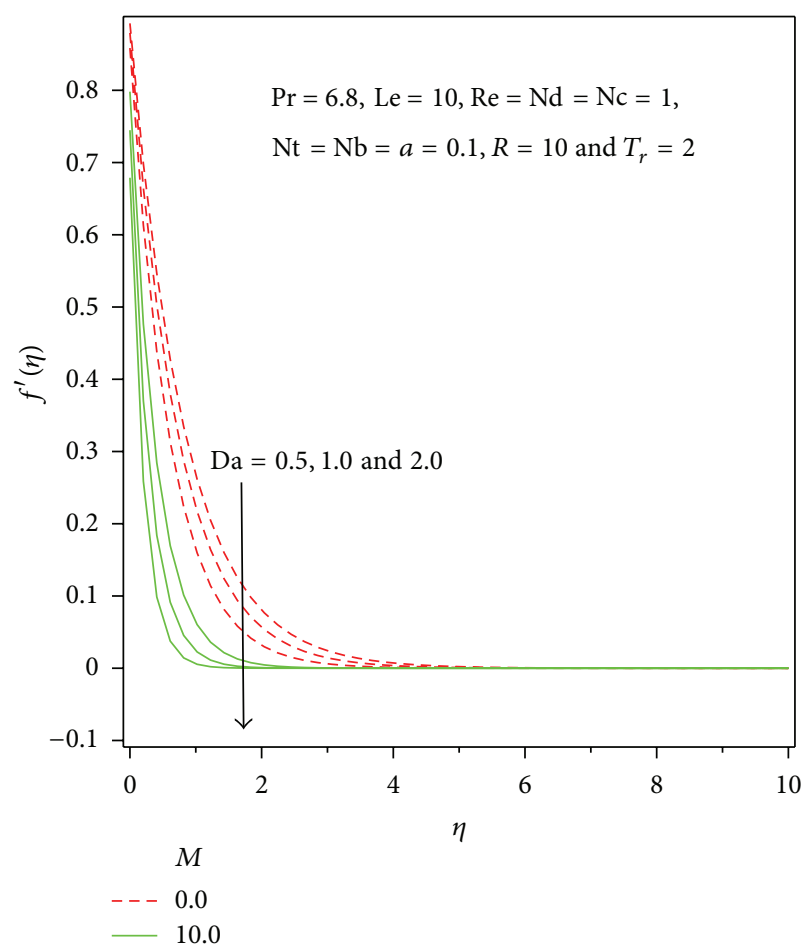

Figure 4: Effect of Darcy number on temperature for various magnetic parameters.

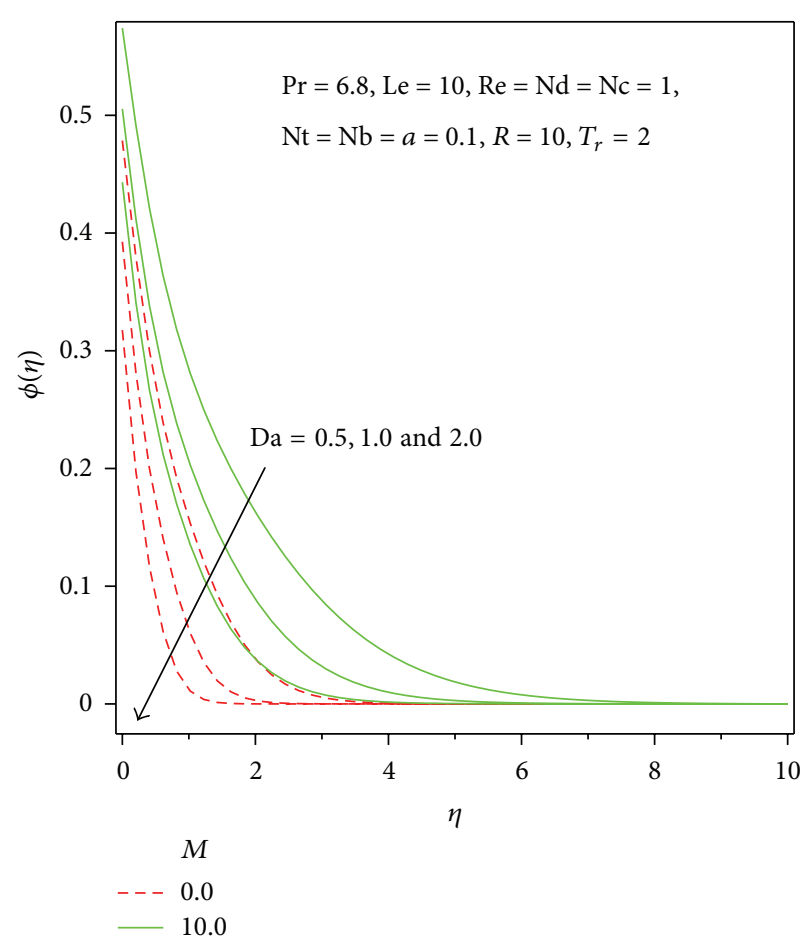

FIGURE 5: Effect of Darcy number on concentration for various magnetic parameters. 


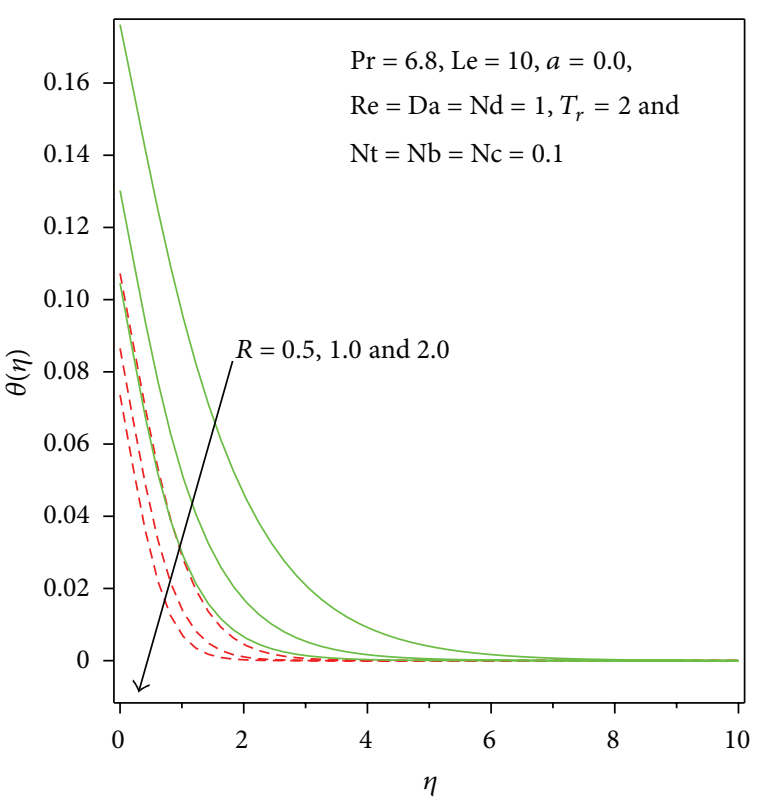

$M$

$---0.0$

10.0

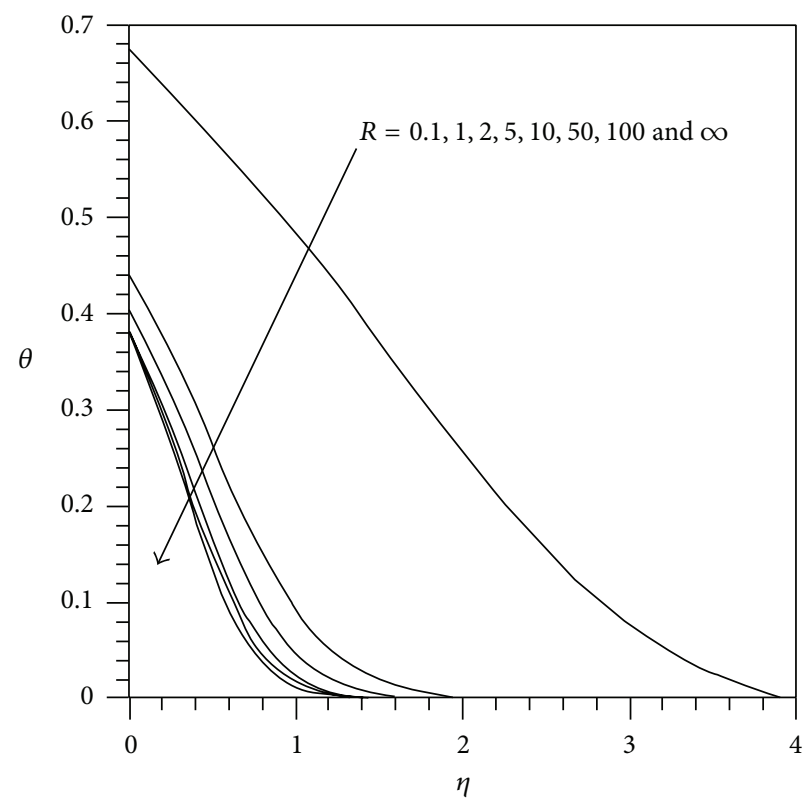

(a)

(b)

FIGURE 6: (a) Effect of conduction-radiation parameter on temperature for various magnetic parameters. (b) Effect of conduction-radiation parameter on temperature profiles [39].

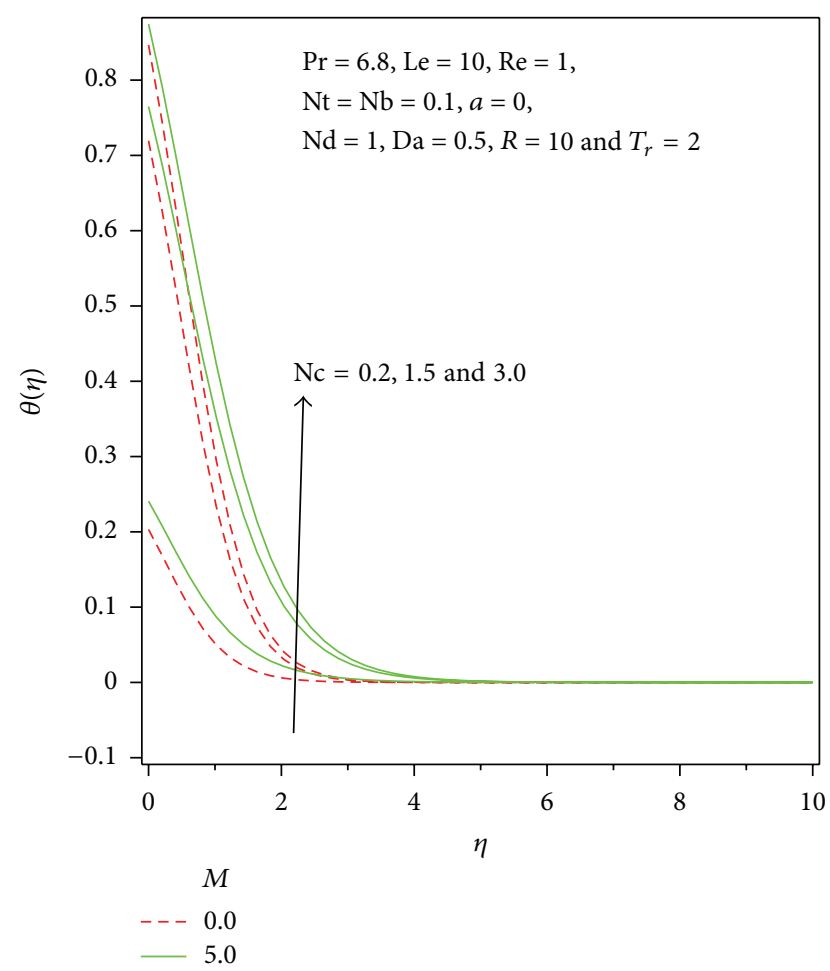

FIGURE 7: Effect convection-conduction parameter on temperature for various magnetic parameters.

Figure 1 shows the variation of the velocity through the boundary layer with momentum slip parameter $(a)$ and

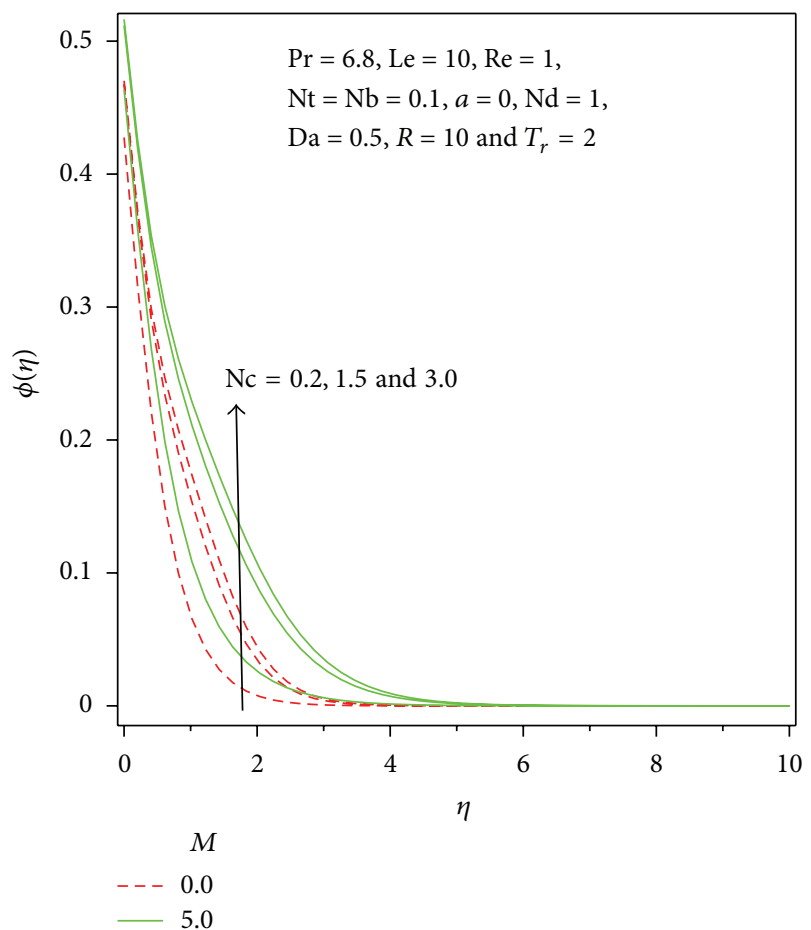

FIGURE 8: Effect convection-conduction parameter on concentration for various magnetic parameters.

various magnetic field parameters $(M)$. It is found that the dimensionless velocity is decreased as the velocity slip parameter increases for both in the presence and absence 


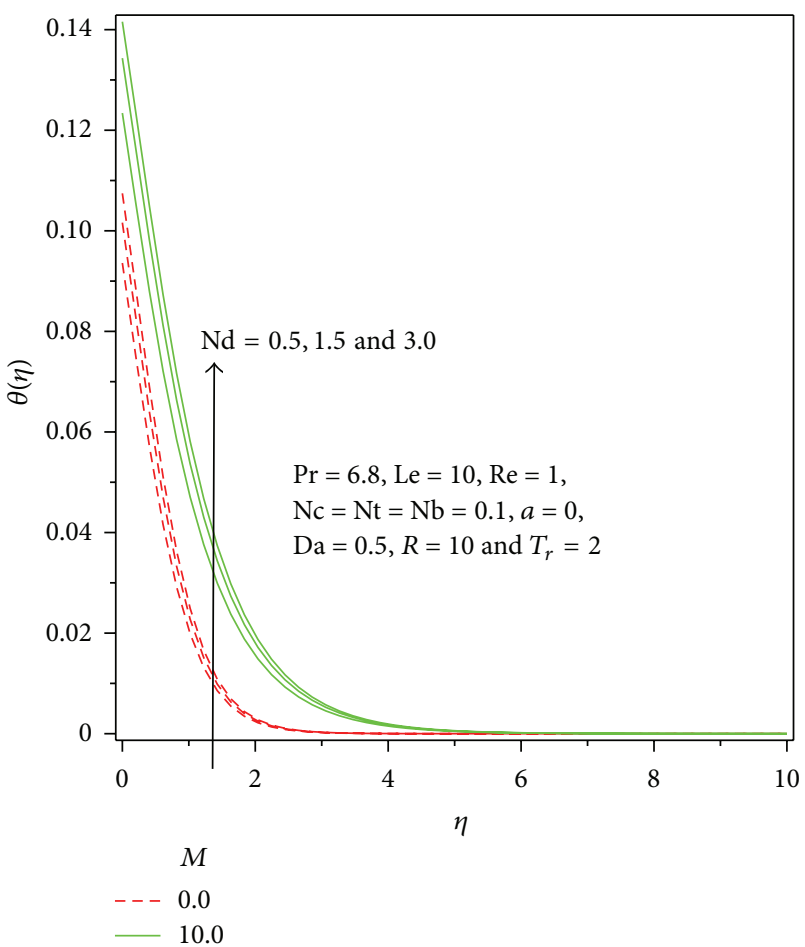

FIGURE 9: Effect convection-diffusion parameter on temperature for various magnetic parameters.

of magnetic field. Physically, the velocity slipping parameter $a$ enhances the differences between the wall and the fluid velocities adjacent to the wall increases. Further, it is seen that, as the slipping parameter enhances, the thickness of the velocity boundary layer decreases. Increasing the slipping factor may be looked at as a miscommunication between the source of motion (the plate) and the fluid domain. It is clear that hydrodynamic behavior of the problem under consideration is more sensitive to the variations in small values of $a$ as compared with the variations in large values of $a$. Note that the case $a=0$ corresponds to the conventional no slip boundary condition at the wall. There is a strong decrease in velocity with magnetic filed as well as slip parameters. Clearly the presence of a magnetic field retards the flow for both slip flow and nonslip flow. An application of a transverse magnetic field to an electrically conducting fluid gives rise to a resistive type force known as the Lorentz force. This force has the tendency to retard the motion of the fluid in the boundary layer and to increase its temperature and concentration. The presence of the Lorentzian hydromagnetic drag impedes boundary layer flow strongly and serves as a potent control mechanism. Momentum boundary layer thickness is greatly reduced with strong magnetic field and also strong momentum slip. Asymptotic convergence of all profiles is observed with greater transverse coordinate, testifying to the accurate imposition of infinity boundary conditions.

Figure 2 demonstrates that, with an increase in momentum slip parameter, the temperature is substantially elevated throughout the boundary layer. Physically, as the velocity slip parameter $a$ increases, less flow will be induced close to the

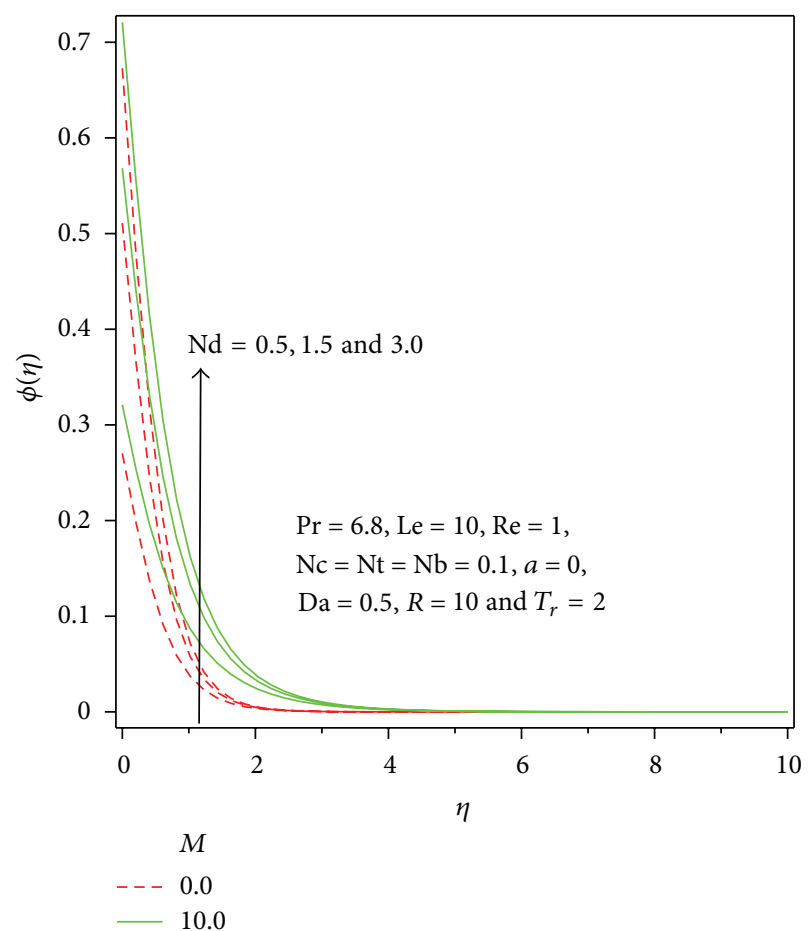

FIGURE 10: Effect of convection-diffusion parameter on concentration for various magnetic parameters.

plate layer and, hence, the hot plate heats a lesser amount of fluid and this causes higher increases in the fluid temperature. Temperatures are also observed to be markedly higher with strong magnetic field $(M=10.0)$ as compared with an absence of magnetic field $(M=0.0)$. Greater momentum slip clearly aids in thermal diffusion from the wall to the body of the fluid, which results in a heating of the boundary layer and significant increase in thermal boundary layer thickness. With magnetic field being present, Lorentzian magnetic body force (see (5)) necessitates greater work from the fluid to sustain motion. This supplementary work needed to drag the fluid against the magnetic field is dissipated as thermal energy (heat) and thermal boundary layer thickness is therefore elevated. With strong momentum slip and magnetic field ( $a=0.8 ; M=10.0$ ), thermal boundary layer thickness is maximum with the converse apparent for the nonslip and nonconducting case $(a=0, M=0)$. The presence of strong wall slip and magnetic field therefore significantly alters the temperature distribution in the regime and this is important in manipulating material characteristics in processing operations.

Figure 3 also demonstrates that increasing momentum slip and magnetic field serves to enhance the nanoparticle concentration. Concentration boundary layer thickness will therefore be strongly increased with greater wall slip and magnetic field strength. With wall slip being absent, concentration magnitudes are clearly observed to be reduced. Generally Figures 1-3 show that momentum diffusion is inhibited with hydrodynamic slip and magnetic field whereas energy and species (nanoparticle concentration) diffusion are aided. 
Figures 4 and 5 illustrate the temperature and nanoparticle concentration response to a variation in Darcy and magnetic field parameters. Physically, greater Darcy number implies a greater permeabiity in the porous medium. This corresponds to a decrease in presence of solid fibers and a reduction in thermal conduction heat transfer within the medium. Increasing Da values will therefore result in a fall in temperatures in the regime, as clearly observed in Figure 4. This will be accompanied by a decrease in thermal boundary layer thickness. Similarly Figure 5 shows that concentration magnitudes are depressed with greater Darcy number, and this results in a decrease in concentration boundary layer thickness. The negative effect of increasing magnetic field on both temperatures and concentrations is confirmed in Figures 4 and 5. Effectively the presence of a porous medium and magnetic field may be exploited to regulate temperature and nanoparticle distributions which is of considerable advantage in the manufacture of nanomaterials where specific spatial properties may be required for different technological applications, as elaborated by Rana et al. [40].

The thermal radiation features in the energy conservation equation (6) are inversely proportional to the conductionradiation parameter $R$. Thus, small $R$ signifies a large radiation effect while $R \rightarrow \infty$ corresponds to no radiation effect. The effect of conduction-radiation parameter (nonlinear Rosseland parameter, $R$ ) on the dimensionless temperature evolution in the boundary layer is shown in Figure 6(a). It represents the relative contribution of thermal conduction heat transfer to thermal radiation heat transfer and when it assumes unity value, both heat transfer modes contribute equally. For $R>1$, conduction will dominate and vice versa for $R<1$. Clearly as $R$ is increased, thermal radiation contribution is depressed and this is manifest with a considerable depletion in temperatures in the boundary layer. Therefore, for low values of $R$, thermal radiation is strong and this will correspond to maximum values of temperature and thicker thermal boundary layers. Evidently the presence of strong thermal radiation flux is demonstrated to heat the thermal boundary layer markedly and is beneficial to materials processing systems where higher temperatures are often required to alter material characteristics. The trends in the present computations have been verified by many other studies in the literature including Rahman and Eltayeb [39], Das [41], Pantokratoras and Fang [42], and Mushtaq et al. [43]. In order to further verify the present numerical method, we compare our Figure 6(a) with Rahman and Eltayeb [39] (see Figure 6(b)). A close agreement is found between present figure and published figure.

Figures 7 and 8 depict the influence of the convectionconduction parameter ( $\mathrm{Nc}$ ) (essentially the Biot number) on the dimensionless temperature and nanoparticle concentration profiles. This parameter arises only in the wall thermal boundary condition (8). It is worth mentioning that the convection-conduction parameter, $\mathrm{Nc}$, is the ratio of the internal thermal resistance of a solid to the boundary layer thermal resistance. When $\mathrm{Nc}=0$ (i.e., without Biot number) the left side of the plate with hot fluid is totally insulated, the internal thermal resistance of the plate is extremely high, and no convective heat transfer to the cold fluid on the right side of the plate takes place. Here the results for constant wall temperature case $\theta(0)=1$ can be recovered when $\mathrm{Nc} \rightarrow \infty$. It exerts a substantial influence on temperature evolution (Figure 7), in particular, as anticipated, at the wall. As Nc is increased, there is a strong elevation in wall temperature values. The larger values of Nc accompany the stronger convective heating at the sheet which rises the temperature gradient at the sheet. This allows the thermal effect to penetrate deeper into the quiescent fluid. Due to this reason the temperature and thermal boundary layer thickness are increasing functions of Nc. The magnitudes with magnetic field being present are also considerably higher than with magnetic field being absent. Thermal boundary layer thickness is therefore minimized with low values of the convection-conduction parameter. The concentration field is driven by the temperature gradient and since temperature is an increasing function of $\mathrm{Nc}$, one would expect an increase in $\phi$ with an increase in Nc. This is what we can observe from Figure 8. Concentration boundary layer thickness is therefore also enhanced with increasing convection-conduction parameter.

Figures 9 and 10 display the effect of the convectiondiffusion parameter $(\mathrm{Nd})$ on temperature and nanoparticle concentration (species) distributions, respectively. This parameter also features only in a single boundary condition, namely, the wall concentration gradient boundary condition in (8), and is inversely proportional to the Brownian diffusion coefficient. A strong enhancement in both temperatures and concentration values is generated with increasing $\mathrm{Nd}$ values. Both thermal and concentration boundary layer thickness are therefore enhanced. With magnetic being field present, magnitudes of temperature and nanoparticle concentration are always greater than for the electrically nonconducting case $(M=0)$. As with the Nc parameter, the most significant response in temperature and nanoparticle concentration profiles is witnessed at the wall, since both parameters are simulated via wall boundary conditions.

\section{Conclusions}

A mathematical model has been developed for steady-state two-dimensional incompressible laminar nanofluid magnetohydrodynamic convective-radiative flow in a porous medium adjacent to a vertical sheet. The left of the sheet is heated by the convection from a hot fluid which provides a variable heat transfer coefficient leading to the presence of a thermal convective boundary condition. Hydrodynamic slip and thermal radiation heat transfer have been included in the model and furthermore a mass convective boundary condition is incorporated. The transformed momentum, energy, and nanoparticle concentration boundary layer equations have been shown to feature a number of thermophysical parameters, namely, Reynolds number, Darcy number, Prandtl number, Lewis number, magnetic field parameter, thermophoresis parameter, Brownian motion parameter, hydrodynamic (momentum) slip parameter, convection-conduction parameter, convection-diffusion parameter, and conduction-radiation parameter (nonlinear 
Rosseland parameter). A Maple numerical solution employing Runge-Kutta-Fehlberg quadrature has been obtained for the strongly nonlinear boundary value problem. The effects of several parameters on the momentum, heat, and mass transfer (species diffusion) characteristics of the nanofluid have been evaluated in detail. Computations have also been validated with earlier published results, demonstrating very good correlation. The present solutions have shown the follwoing.

(i) Increasing hydrodynamic (momentum) slip significantly retards the boundary layer flow, whereas it markedly increases temperature and nanoparticle concentration values.

(ii) Increasing magnetic field damps the velocity characteristics, that is, decelerates the flow, whereas it enhances both temperatures and nanoparticle concentration values.

(iii) Increasing Darcy number (corresponding to greater porous medium permeability) strongly boosts the temperature and concentration magnitudes.

(iv) Decreasing Rosseland conduction-radiation parameter (which corresponds to greater thermal radiative heat flux presence) notably elevates temperatures in the boundary layer.

(v) Increasing convection-conduction parameter (Nc) (which is simulated via a thermal convective boundary condition) strongly enhances both temperature and nanoparticle concentration values.

(vi) Increasing convection-diffusion parameter $(\mathrm{Nd})$ (which is simulated via a mass convective boundary condition) strongly enhances both temperature and nanoparticle concentration values.

The present study has considered Newtonian steady-state magnetoconvective nanofluid flow. Transient [44] and nonNewtonian effects [45] are also relevant to magnetic nanomaterials processing operations, and these will be considered in the near future.

\section{Nomenclature}

$\begin{array}{ll}a: & \text { Slip parameter } \\ B_{0}: & \text { Constant magnetic field } \\ C: & \text { Dimensional concentration } \\ c_{p}: & \text { Specific heat at constant pressure } \\ & (\mathrm{J} / \mathrm{kg} \mathrm{K}) \\ C_{f \bar{x}}: & \text { Skin friction factor } \\ D_{B}: & \text { Brownian diffusion coefficient } \\ D_{T}: & \text { Thermophoretic diffusion coefficient } \\ f(\eta): & \text { Dimensionless stream function } \\ h_{f}(\bar{x}): & \text { Local heat transfer coefficient }\left(\mathrm{W} / \mathrm{m}^{2} \mathrm{~K}\right) \\ \left(h_{f}\right)_{0}: & \text { Constant heat transfer coefficient } \\ h_{m}(\bar{x}): & \text { Local mass transfer coefficient } \\ \left(h_{m}\right)_{0}: & \text { Constant mass transfer coefficient } \\ k: & \text { Thermal conductivity }\left(\mathrm{m}^{2} / \mathrm{s}\right) \\ L: & \text { Characteristic length of the plate }(\mathrm{m})\end{array}$

Le: Lewis number

M: $\quad$ Magnetic field parameter

$N_{1}(\bar{x})$ : Variable velocity slip factor

$\mathrm{Nb}$ : Brownian motion parameter

Nc: Conduction-convection parameter

Nd: Diffusion-convection parameter

Nt: Thermophoresis parameter

$\mathrm{Nu}_{\bar{x}}: \quad$ Heat transfer rate

Pr: $\quad$ Prandtl number

$p: \quad$ Pressure $(\mathrm{Pa})$

$q_{m}: \quad$ Wall mass flux $\left(\mathrm{kg} / \mathrm{s} \mathrm{m}^{2}\right)$

$q_{w}$ : Wall heat flux $\left(\mathrm{W} / \mathrm{m}^{2}\right)$

Re: $\quad$ Reynolds number

$\mathrm{Re}_{\bar{x}}: \quad$ Local Reynolds number

A: $\quad$ Thermal conductivity parameter

$\mathrm{Sh}_{\bar{x}}: \quad$ Local Sherwood number

$T: \quad$ Dimensional temperature

$\bar{u}, \bar{v}: \quad$ Velocity components along axes

$\bar{u}_{e}: \quad$ External velocity $(\mathrm{m} / \mathrm{s})$

$U_{\infty}: \quad$ Reference velocity $(\mathrm{m} / \mathrm{s})$

$\bar{x}, \bar{y}$ : Cartesian coordinates aligned along and normal to the plate $(\mathrm{m})$.

Greek Symbols

$\alpha: \quad$ Thermal diffusivity $\left(\mathrm{m}^{2} / \mathrm{s}\right)$

$\alpha_{i}: \quad$ Real numbers

$\beta$ : $\quad$ Pressure gradient parameter

$\tau$ : $\quad$ Ratio of effective heat capacity of the nanoparticle material to the heat capacity of the fluid

$\sigma: \quad$ Electric conductivity

$\theta(\eta)$ : Dimensionless temperature

$\phi(\eta)$ : Dimensionless concentration (nanoparticle volume fraction)

$\eta$ : Similarity variable

$\mu$ : Dynamic viscosity of the fluid $\left(\mathrm{Ns} / \mathrm{m}^{2}\right)$

$v$ : Kinematic viscosity of the fluid $\left(\mathrm{m}^{2} / \mathrm{s}\right)$

$\rho_{f}: \quad$ Fluid density $\left(\mathrm{kg} / \mathrm{m}^{3}\right)$

$\psi$ : Stream function.

Subscripts

$w$ : Condition at the wall

$\infty$ : Ambient condition.

\section{Conflict of Interests}

The authors declare that there is no conflict of interests regarding the publication of this paper.

\section{Acknowledgment}

The authors acknowledge financial support from Universiti Sains Malaysia, RU Grant 1001/PMATHS/811252.

\section{References}

[1] S. Choi, "Enhancing thermal conductivity of fluids with nanoparticles," in Developments and Applications of Non-Newtonian 
Flows, Presented at the ASME International Mechanical Engineering Congress and Exposition, November 12-17,San Francisco, Calif, USA, Volume 231, 1995, D. A. Siginer and H. P. Wang, Eds., vol. 66, pp. 99-105, ASME, 1995.

[2] P. Rana, R. Bhargava, and O. A. Bég, "Numerical solution for mixed convection boundary layer flow of a nanofluid along an inclined plate embedded in a porous medium," Computers \& Mathematics with Applications, vol. 64, no. 9, pp. 2816-2832, 2012.

[3] P. Keblinski, "Nanofluids for enhanced thermal transport: understanding and controversy," in Proceedings of the Symposium II Nanoscale Heat Transport-From Fundamentals to Devices Materials Research Society Spring Symposium, San Francisco, Calif, USA, April 2007.

[4] O. A. Bég, V. R. Prasad, and B. Vasu, "Numerical study of mixed bioconvection in porous media saturated with nanofluid and containing oxytactic micro-organisms," Journal Mechanics Medicine Biology, vol. 13, no. 4, Article ID 1350067, 25 pages.

[5] M. J. Uddin, N. H. Md. Yusoff, O. A. Bég, and A. I. M. Ismail, "Lie group analysis and numerical solutions for non-Newtonian nanofluid flow in a porous medium with internal heat generation," Physica Scripta, vol. 87, Article ID 025401, 14 pages, 2013.

[6] M. M. Rashidi, O. A. Bég, M. Asadi, and M. T. Rastegari, "DTMPadé modeling of natural convective boundary layer flow of a nanofluid past a vertical surface," International Journal Thermal Environmental Engineering, vol. 4, pp. 13-24, 2012.

[7] D. Tripathi and O. A. Bég, "A study on peristaltic flow of nanofluids: application in drug delivery systems," International Journal of Heat and Mass Transfer, vol. 70, pp. 61-70, 2014.

[8] T. A. Bég, O. A. Bég, M. M. Rashidi, and M. Asadi, "Homotopy semi-numerical modelling of nanofluid convection flow from an isothermal spherical body in a permeable regime," International Journal of Microscale and Nanoscale Thermal and Fluid Transport Phenomena, vol. 3, pp. 67-96, 2012.

[9] J. Buongiorno, "Convective transport in nanofluids," Journal of Heat Transfer, vol. 128, no. 3, pp. 240-250, 2006.

[10] D. A. Nield and A. V. Kuznetsov, "The onset of double-diffusive convection in a nanofluid layer," International Journal of Heat and Fluid Flow, vol. 32, no. 4, pp. 771-776, 2011.

[11] H. Nemati, M. Farhadi, K. Sedighi, H. R. Ashorynejad, and E. Fattahi, "Magnetic field effects on natural convection flow of nanofluid in a rectangular cavity using the Lattice Boltzmann model," Scientia Iranica, vol. 19, no. 2, pp. 303-310, 2012.

[12] M. A. A. Hamad and I. Pop, "Unsteady MHD free convection flow past a vertical permeable flat plate in a rotating frame of reference with constant heat source in a nanofluid," Heat and Mass Transfer, vol. 47, no. 12, pp. 1517-1524, 2011.

[13] S. M. Aminossadati, A. Raisi, and B. Ghasemi, "Effects of magnetic field on nanofluid forced convection in a partially heated microchannel," International Journal of Non-Linear Mechanics, vol. 46, no. 10, pp. 1373-1382, 2011.

[14] S. Kadri, R. Mehdaoui, and M. Elmi, "Vertical magnetoconvection in square cavity containing a $\mathrm{Al} 2 \mathrm{O} 3$ + water nanofluid: cooling of electronic compounds," Energy Procedia, vol. 18, pp. 724-732, 2012.

[15] T. R. Mahapatra, S. K. Nandy, and A. S. Gupta, "Oblique stagnation-point flow and heat transfer towards a shrinking sheet with thermal radiation," Meccanica, vol. 47, no. 6, pp. 13251335, 2012.

[16] J. Zueco and O. A. Bég, "Network simulation solutions for laminar radiating dissipative magneto-gas dynamic heat transfer over a wedge in non-Darcian porous regime," Mathematical and Computer Modelling, vol. 50, no. 3-4, pp. 439-452, 2009.

[17] K. Vafai and C.-L. Tien, "Convective and radiative heat transfer in porous media," Advances in Applied Mechanics, vol. 27, pp. 225-282, 1989.

[18] H. S. Takhar, O. A. Bèg, and M. Kumari, "Computational analysis of coupled radiation-convection dissipative non-gray gas flow in a non-darcy porous medium using the keller-box implicit difference scheme," International Journal of Energy Research, vol. 22, no. 2, pp. 141-159, 1998.

[19] M. M. Rashidi, M. T. Rastegari, and O. A. Bég, "Homotopy analysis of Soret and Dufour effects on free convection nonNewtonian flow in a porous medium with thermal radiation flux," International Journal Applied Mathematics and Mechanics, vol. 9, no. 2, pp. 39-368, 2013.

[20] D. A. Nield and A. V. Kuznetsov, "The Cheng-Minkowycz problem for natural convective boundary-layer flow in a porous medium saturated by a nanofluid," International Journal of Heat and Mass Transfer, vol. 52, no. 25-26, pp. 5792-5795, 2009.

[21] Md. J. Uddin, W. A. Khan, and A. I. Md. Ismail, "Lie group analysis of natural convective flow from a convectively heated upward facing radiating permeable horizontal plate in porous media filled with nanofluid," Journal of Applied Mathematics, vol. 2012, Article ID 648675, 18 pages, 2012.

[22] F. M. Hady, F. S. Ibrahim, S. M. Abdel-Gaied, and M. R. Eid, "Boundary-layer non-Newtonian flow over vertical plate in porous medium saturated with nanofluid," Applied Mathematics and Mechanics, vol. 32, no. 12, pp. 1577-1586, 2011.

[23] O. A. Bég, T. A. Bég, M. M. Rashidi, and M. Asadi, "DTMPadé semi-numerical simulation of nanofluid transport in porous media," International Journal of Applied Mathematics and Mechanics, vol. 9, pp. 10-32, 2013.

[24] R. Kandasamy, I. Muhaimin, N. S. Ram, and K. K. S. Prabhu, "Thermal stratification effects on Hiemenz flow of nanofluid over a porous wedge sheet in the presence of suction/injection due to solar energy: lie group transformation," Transport in Porous Media, vol. 94, no. 1, pp. 399-416, 2012.

[25] R. S. R. Gorla and A. Chamkha, "Natural convective boundary layer flow over a nonisothermal vertical plate embedded in a porous medium saturated with a nanofluid," Nanoscale and Microscale Thermophysical Engineering, vol. 15, no. 2, pp. 81-94, 2011.

[26] M. H. Yasin, N. M. Arifin, R. Nazar R, F. Ismail, and I. Pop, "Mixed convection boundary layer with internal heat generation in a porous medium filled with a nanofluid," Advanced Science Letters, vol. 13, pp. 833-835, 2012.

[27] O. D. Makinde and A. Aziz, "Boundary layer flow of a nanofluid past a stretching sheet with a convective boundary condition," International Journal of Thermal Sciences, vol. 50, no. 7, pp. 13261332, 2011.

[28] M. A. A. Hamad, M. J. Uddin, and A. I. M. Ismail, "Investigation of combined heat and mass transfer by Lie group analysis with variable diffusivity taking into account hydrodynamic slip and thermal convective boundary conditions," International Journal of Heat and Mass Transfer, vol. 55, no. 4, pp. 1355-1362, 2012.

[29] V. R. Prasad, A. Subba Rao, N. Bhaskar Reddy, B. Vasu, and O. A. Bég, "Modelling laminar transport phenomena in a Casson rheological fluid from a horizontal circular cylinder with partial slip," Proceedings of the Institution of Mechanical Engineers E: Journal of Process Mechanical Engineering, 2012. 
[30] V. R. Prasad, A. S. Rao, N. B. Reddy, and O. A. Bég, "Heat transfer in a Casson rheological fluid from a semi-infinite vertical plate with partial slip," Heat Transfer-Asian Research, 2014.

[31] V. R. Prasad, A. S. Rao, N. B. Reddy, and O. A. Bég, "Modelling laminar transport in a Casson fluid from an isothermal sphere with partial slip in a non-Darcy porous medium," Theoretical Applied Mechanics, vol. 40, no. 4, 2013.

[32] A. Aziz, Heat Conduction with Maple, CRC Press, New York, NY, USA, 2005.

[33] M. Dayyan, S. M. Seyyedi, G. G. Domairry, and M. Gorji Bandpy, "Analytical solution of flow and heat transfer over a permeable stretching wall in a porous medium," Mathematical Problems in Engineering, vol. 2013, Article ID 682795, 10 pages, 2013.

[34] A. K. Datta, Biological and Bioenvironmental Heat and Mass Transfer, Marcel Dekker, New York, NY, USA, 2002.

[35] G. Karniadakis, A. Beskok, and N. Aluru, Microflows and Nanoflows Fundamentals and Simulation, Springer Science, New York, NY, USA, 2005.

[36] R. Cortell, "Effects of viscous dissipation and radiation on the thermal boundary layer over a nonlinearly stretching sheet," Physics Letters A: General, Atomic and Solid State Physics, vol. 372, no. 5, pp. 631-636, 2008.

[37] O. A. Bég, J. Zueco, T. A. Bég, H. S. Takhar, and E. Kahya, "NSM analysis of time-dependent nonlinear buoyancy-driven doublediffusive radiative convection flow in non-Darcy geological porous media," Acta Mechanica, vol. 202, no. 1-4, pp. 181-204, 2009.

[38] E. M. Sparrow and R. D. Cess, Radiation Heat Transfer, chapters 7 \& 10, Hemisphere, Washington, DC, USA.

[39] M. M. Rahman and I. A. Eltayeb, "Radiative heat transfer in a hydromagnetic nanofluid past a non-linear stretching surface with convective boundary condition," Meccanica, vol. 48, no. 3 , pp. 601-615, 2013.

[40] P. Rana, R. Bhargava, and O. A. Bég, "Finite element simulation of unsteady MHD transport phenomena on a stretching sheet in a rotating nanofluid," in Proceedings of the Institution of Mechanical Engineers N: Journal of Nanoengineering and Nanosystems, vol. 227, pp. 277-299, 2013.

[41] K. Das, "Slip effects on heat and mass transfer in MHD micropolar fluid flow over an inclined plate with thermal radiation and chemical reaction," International Journal for Numerical Methods in Fluids, vol. 70, no. 1, pp. 96-113, 2012.

[42] A. Pantokratoras and T. Fang, "Sakiadis flow with nonlinear Rosseland thermal radiation," Physica Scripta, vol. 87, Article ID 015703, 5 pages, 2013.

[43] A. Mushtaq, M. Mustafa, T. Hayat, and A. Alsaedi, "Nonlinear radiative heat transfer in the flow of nanofluid due to solar energy: a numerical study," Journal of the Taiwan Institute of Chemical Engineers, 2013.

[44] O. A. Bég, M. S. Khan, I. Karim, M. M. Alam, and M. Ferdows, "Explicit numerical study of unsteady hydromagnetic mixed convective nanofluid flow from an exponentially-stretching sheet in porous media," Applied Nanoscience, 2013.

[45] M. J. Uddin, M. Ferdows, and O. A. Bég, "Group analysis and numerical computation of magneto-convective nonNewtonian nanofluid slip flow from a permeable stretching sheet," Applied Nanoscience, 2013. 


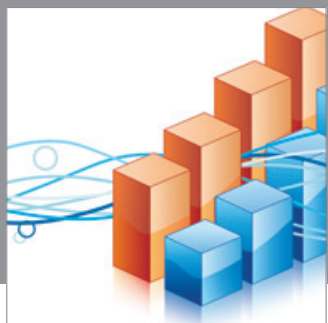

Advances in

Operations Research

mansans

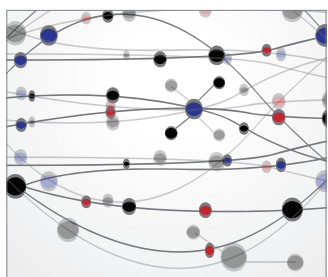

The Scientific World Journal
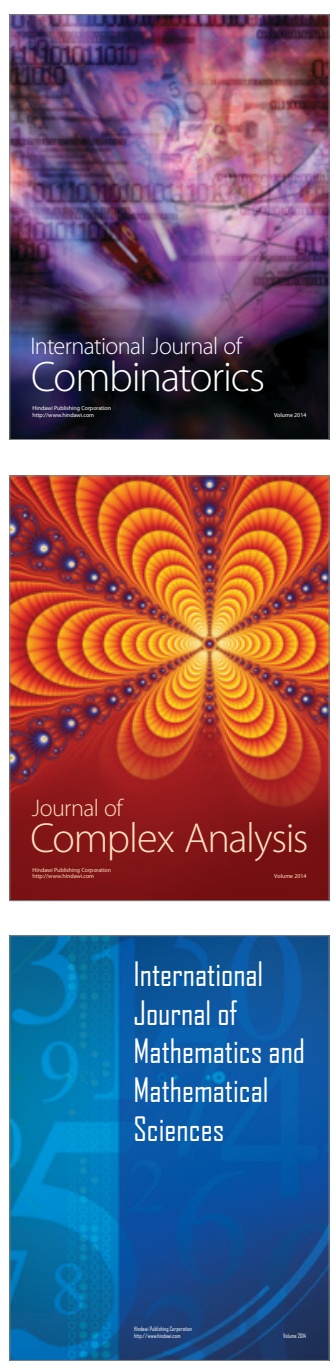
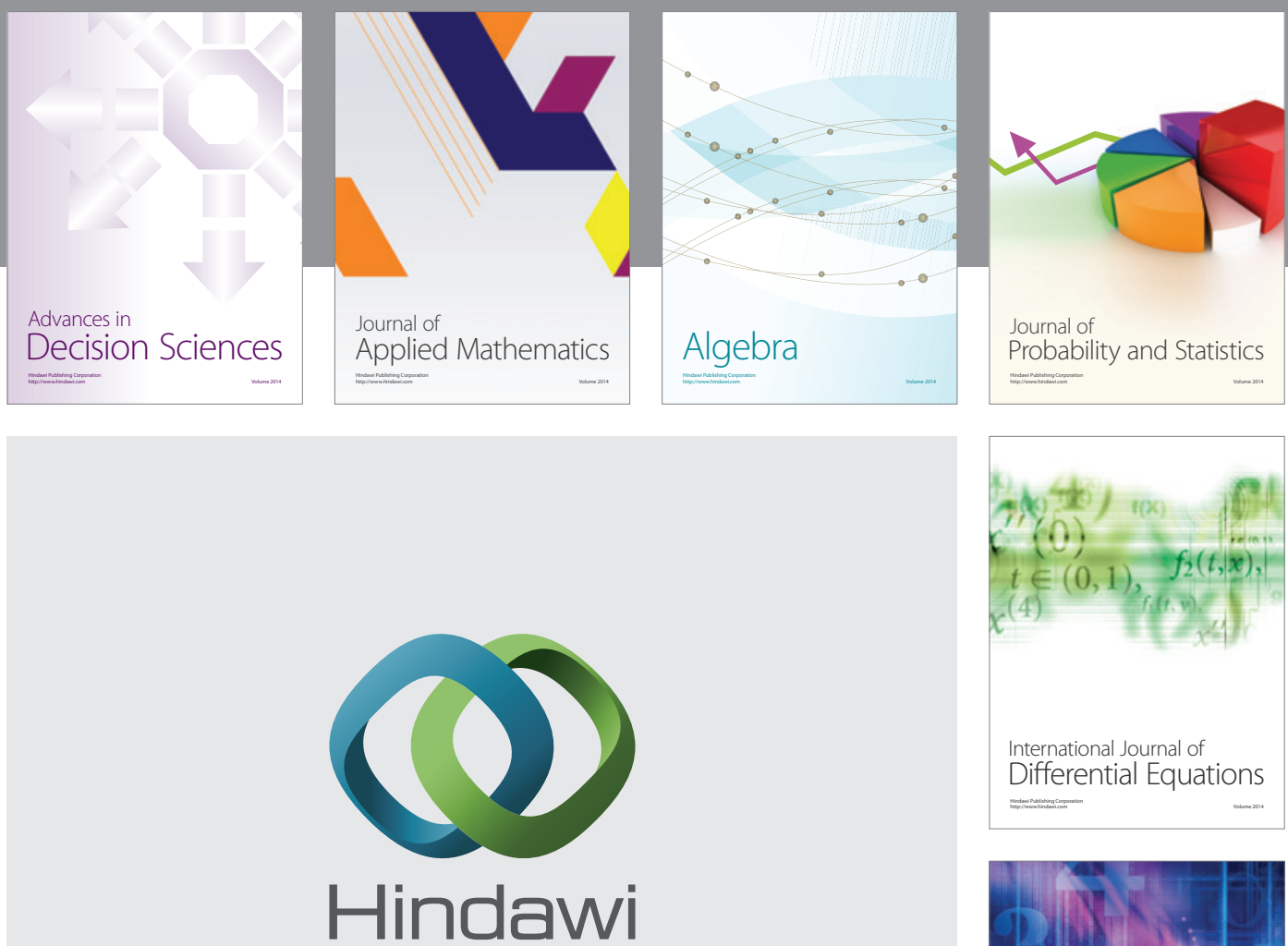

Submit your manuscripts at http://www.hindawi.com
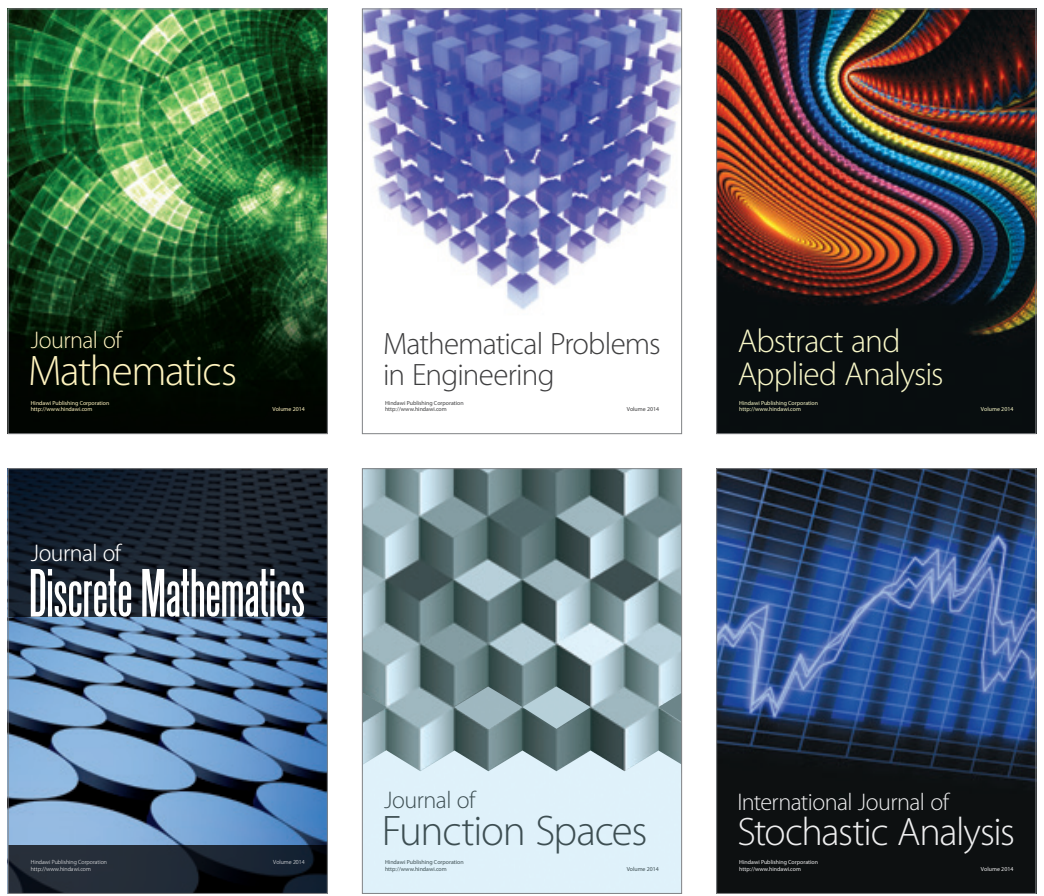

Journal of

Function Spaces

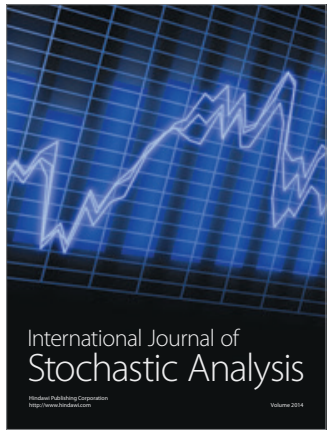

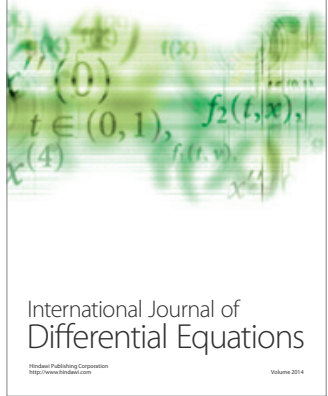
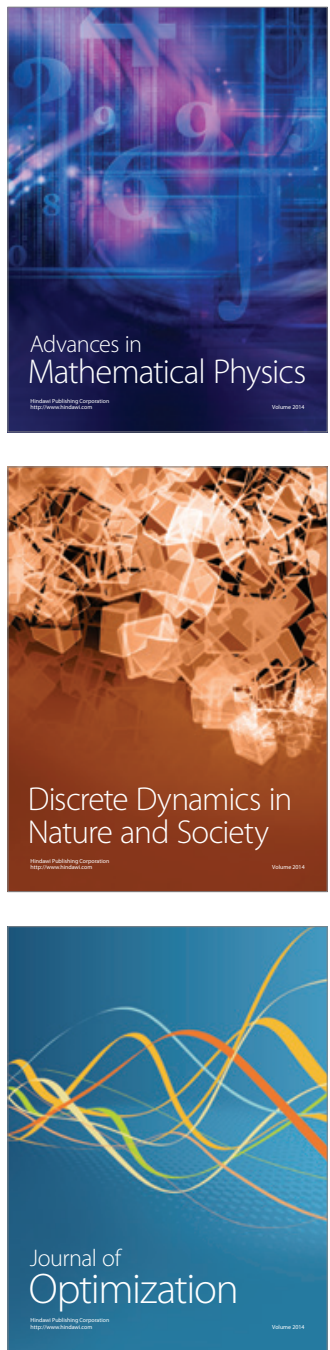